INFORME DE PRÁCTICA EMPRESARIAL

MANEJO DE INVENTARIOS EN LA EMPRESA SYSCON LTDA

SANTIAGO CARDONA OCHOA

ASESOR DE PRACTICA

EDUARDO NICOLÁS CUETO FUENTES

TRABAJO PRESENTADO COMO REQUISITO PARA OPTAR AL TÍTULO PROFESIONAL DE CONTADURÍA PÚBLICA

\author{
UNIVERSIDAD SANTO TOMÁS \\ FACULTAD DE CONTADURÍA PÚBLICA
}

MEDELLÍN

26. NOVIEMBRE. 2018 
TABLA DE CON CONTENIDO

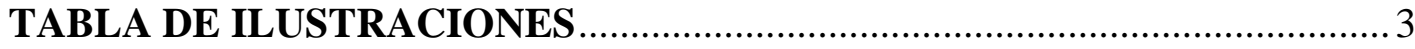

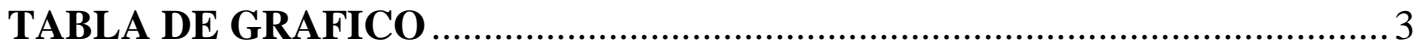

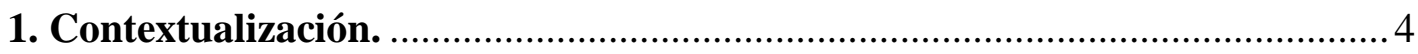

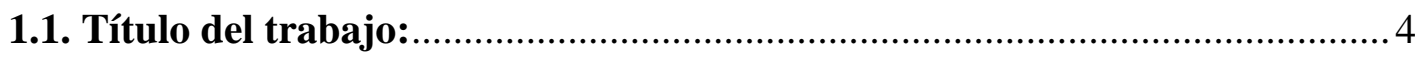

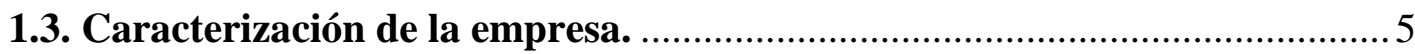

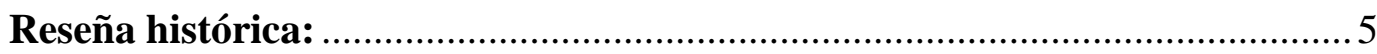

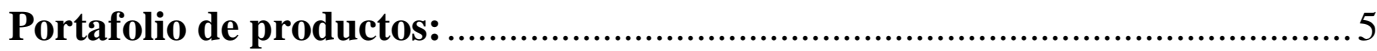

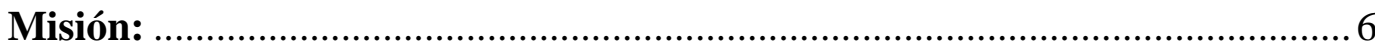

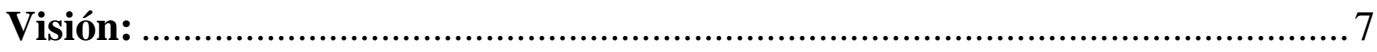

Logotipo:

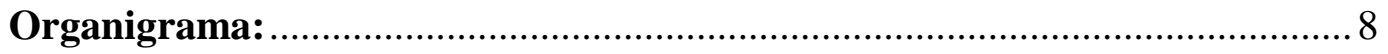

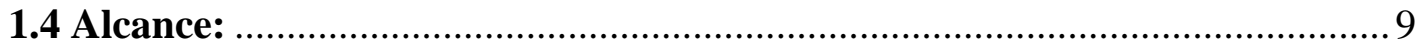

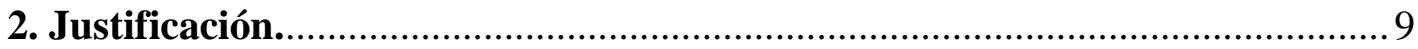

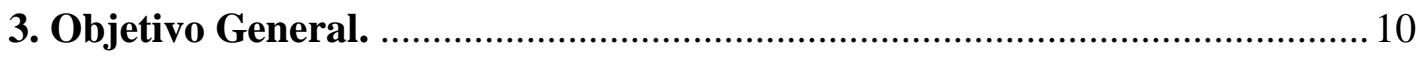

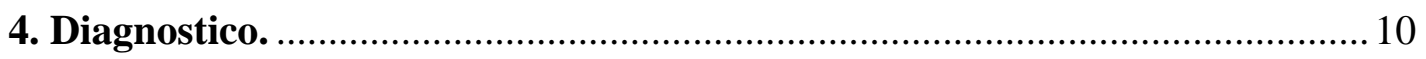

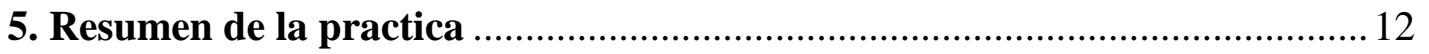

6. Planteamiento del problema ......................................................................... 12

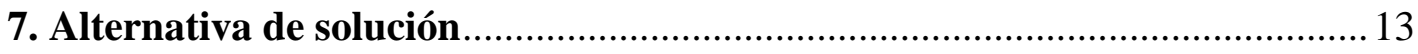

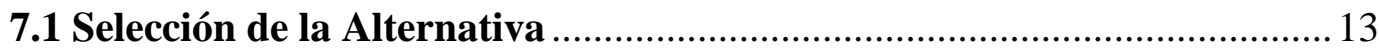

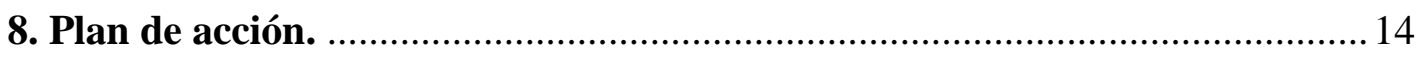

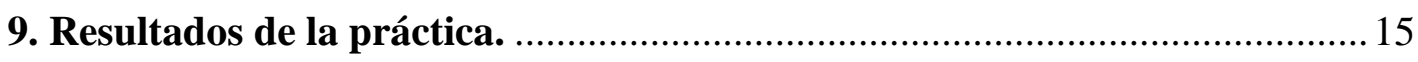

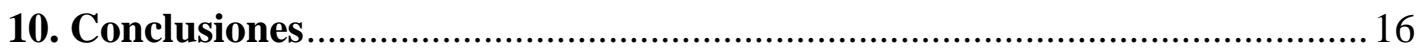

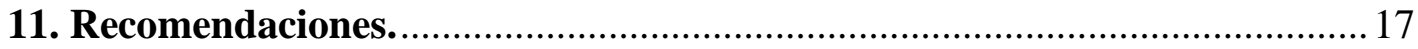

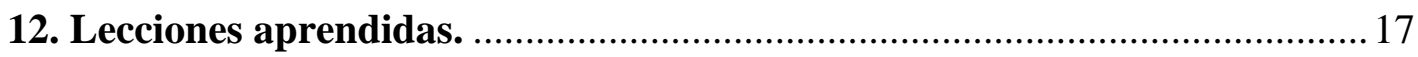

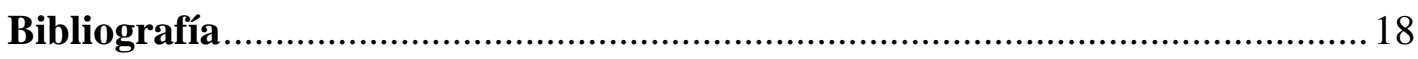

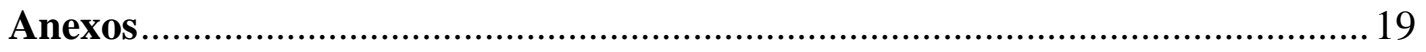




\section{TABLA DE ILUSTRACIONES}

Ilustración 1. Logotipo SYSCON

\section{TABLA DE GRAFICO}

Grafico 1. Organigrama Empresa SYSCON …………........................................ 8

\section{TABLA DE CUADROS}

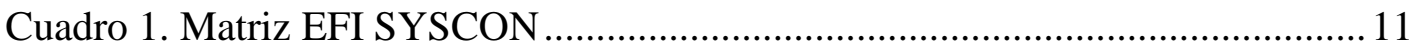

Cuadro 2. Plan de accion. SYSCO ……………………........................................ 14

\section{INTRODUCCION.}

El control de inventario juega un papel muy importante en las empresas, por lo que una mala administración, gestión y control de estas puede ocasionar diversos problemas no solo con los clientes sino también problemas más graves como los financieros.

Sin embargo, no solamente los comercializadores y productores mantienen inventarios. Cada vez es más frecuente y necesario utilizar el concepto de inventarios para los prestadores de servicios. Por esta razón, las Normas Internacionales para Pymes establecen el tratamiento para este tipo de partidas.

"los prestadores de servicios reconocerán como inventarios los costos incurridos en la prestación de servicios, de los cuales no se hayan reconocido aún los ingresos correspondientes" (Herrera, 2016). 
Las empresas prestadoras de servicio pueden tener costos de inventarios procedentes de su actividad de servicios, los costos se conforman principalmente de:

Mano de obra del personal involucrado directamente en la prestación de servicio.

Costo del personal de supervisión y el personal de revisión de acuerdo a los servicios prestados.

Otros costos indirectos atribuibles al servicio, tales como depreciación de equipos, materiales utilizados, honorarios de terceros, servicios técnicos, servicios públicos, entre otros.

Es así como concluimos que es necesario una implementación muy eficiente del control de inventarios sobre todo en las empresas de prestación de servicios, donde existe una delgada línea entre lo que se considera costos de inventario y los costos reales de la empresa, es muy importante definir y discriminar estos dos conceptos para así llegar a tener un manejo más eficiente y de más control sobre estos.

\section{Contextualización.}

\subsection{Título del trabajo:}

Manejo de inventarios en la empresa SYSCON LTDA

\subsection{Nombre de la empresa:}

SYSCON LTDA 


\subsection{Caracterización de la empresa.}

\section{Reseña histórica:}

Syscon es una empresa que nació en el año 2002 con 3 socios en la ciudad envigado, el objetivo con el cual fue creada la empresa era de crear y desarrollar software para parqueaderos y así estos pudieran mantener un control de la cantidad de vehículos que ingresan y salen de ellos. Más de 10 años después de sus inicios y con el ingreso de nuevos sistemas al mercado, syscon se ha expandido no solo se enfoca en parqueaderos como inicialmente empezó, sino que ya podemos encontrar servicios para unidades residenciales, locales comerciales y software diseñados para cada necesidad que el cliente lo necesite, brindando siempre el mejor servicio y las mejores soluciones.

\section{Portafolio de productos:}

Actualmente la empresa presta un servicio diverso de soluciones dependiendo de las necesidades de cada usuario. Podemos encontrar en el portafolio de SYSCON los siguientes productos.

- Desarrollo a la Medida: Su objetivo principal es el desarrollar una solución de software para una necesidad o iniciativa determinada de acuerdo a las especificaciones que el cliente lo solicite

- Syscon Park Web: Software para parqueaderos, es una solución de software diseñada para el manejo eficiente de los procesos de control, gestión y administración en los estacionamientos, grandes, medianos y pequeños. 
- Syscon CAS Empresarial: Es un software para controlar el ingreso de empleados, proveedores, domicilios y visitantes en la empresa.

- Sistema Guarda equipaje: Es un software que permite registrar el equipaje, controlar el tiempo de permanencia y facturar el servicio de acuerdo a las tarifas.

- Outsourcing Informático: Es un servicio que se ofrece a las empresas para optimizar sus proyectos y procesos informáticos.

- Control y Gestión Automático en parqueaderos: Es un sistema de automatización para control de ingreso y salida de vehículos con máquinas dispensadoras y receptora de tarjetas.

- Syscon CAS para unidades residenciales: Es un sistema de control de ingreso para unidades residenciales diseñado para registrar visitantes, proveedores, domicilios, empleados de apartamentos y empresas de servicios.

- Syscon Software administrativo: El software administrativo controla la información del cliente, empleados, proveedores y productos de su empresa, posee además una agenda que permite elaborar, organizar, planear citas y eventos.

\section{Misión:}

Sistemas y conectividad Syscon, presta el servicio de desarrollo y comercialización de software a la medida de los requerimientos de las empresas a nivel nacional y específicamente soluciones de software para parqueaderos, lavautos, control de acceso para 
Unidades Residenciales y Empresarial. Además de sistemas de automatización en parqueaderos.

\section{Visión:}

Sistemas y conectividad Syscon en el 2020, tendrá reconocimiento, mayor posicionamiento en las empresas a nivel nacional en la prestación de servicios y productos informáticos; diferenciándose por la calidad, la cultura de servicios y la tecnología adecuada, para lograr mayor participación en el mercado, y la lealtad al cliente.

\section{Logotipo:}

\section{Ilustración 1. Logotipo SYSCON}

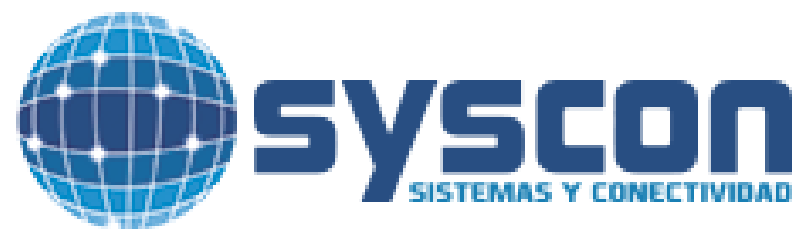

Fuente: Syscon LTDA 


\section{Organigrama:}

Al interior de toda compañía se considera necesario conocer las relaciones que existen entre los elementos que la conforman, así mismo como las posiciones y funciones que realiza cada uno, por lo que resulta necesario entender su estructura organizacional.

Grafico 1. Organigrama Empresa SYSCON

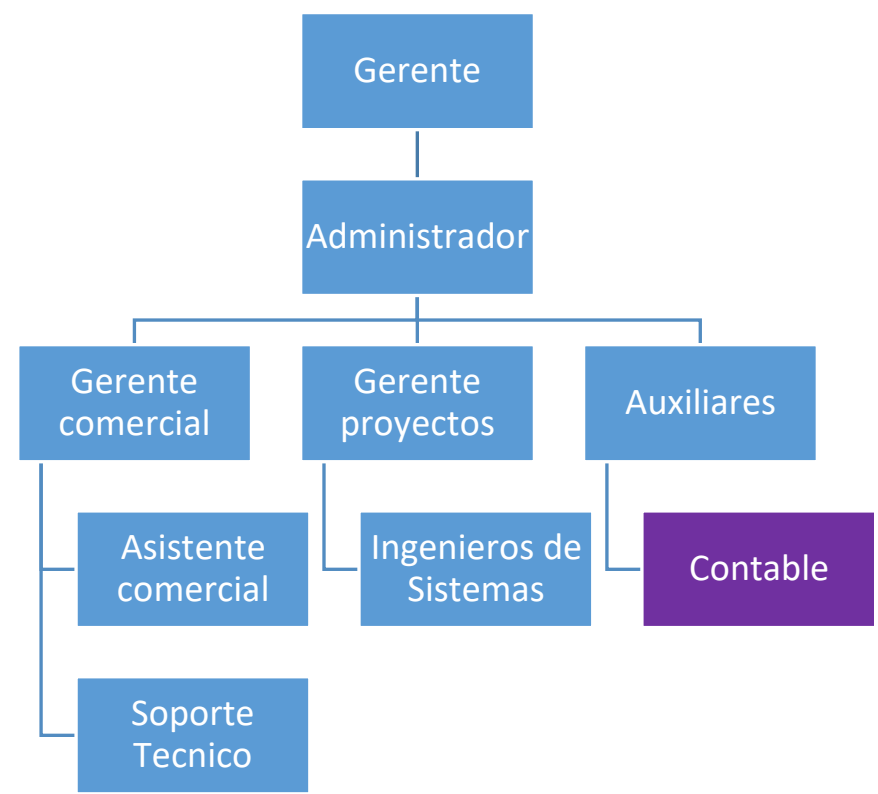

Fuente: elaboración propia, basado en la investigación realizada en la empresa SYSCON

Queda subrayado el área donde se llevará a cabo el desarrollo de las prácticas profesionales, el área contable se encarga básicamente de las siguientes funciones.

- Manejo de la información financiera.

- Manejo de los inventarios, al ser una empresa prestadora de servicios, estos inventarios son el costo al cual incurre la empresa para prestar los servicios.

- Liquidación de los impuestos. 
En la función de manejo de inventarios es donde se lleva a cabo las prácticas formativas, sirviendo de apoyo a todos los procesos que allí se realizan, donde se hará posible el desarrollo de habilidades y conocimientos obtenidos durante la formación profesional en el ámbito de la contaduría pública.

\subsection{Alcance:}

A continuación, se referenciarán los dos principales alcances que se llevarán a cabo durante el desarrollo de la práctica tanto formativa como profesional, y entre los cuales se encuentra:

1. Evaluar y dar un diagnóstico oportuno sobre el control que se ha venido dando a los inventarios, para así tratar de corregir las falencias que ha venido presentando.

2. Darle más importancia a esta área de la empresa la cual al ser una empresa prestadora de servicio no se le brinda la atención adecuada.

\section{Justificación.}

En la empresa SYSCON se han detectado varias irregularidades al momento de hacer un adecuado manejo de inventarios, debido a esto se requiere ofrecer una solución la cual permita mantener un control de inventarios más adecuado y tener un registro permanente de este.

Es muy importante resaltar que un adecuado funcionamiento de los inventarios ayuda a las empresas a una mejor toma de decisiones, tener un control de los costos a los que se incurra (costos de inventarios).

Dada esta situación y con la realización de las practicas al interior de la empresa SYSCON LTDA, se permitirá alcanzar mejor resultados empresariales, económicos, académicos y personales. 


\section{Objetivo General.}

Analizar el manejo de los inventarios, teniendo una adecuada separación entre los costos de inventario y otros costos en la empresa SYSCON LTDA.

\subsection{Objetivos específicos:}

Evaluar el manejo de los inventarios en la empresa

Analizar los problemas que se encontraron para determinar un adecuado manejo Proponer acciones para el mejoramiento del control de los inventarios

\section{Diagnostico.}

A continuación, se referenciará la estructura de la matriz EFI, la construcción de la misma se la misma se elabora a través de la siguiente metodología:

1. Se conforma el grupo focal de la empresa que maneja el acervo de conocimiento sobre la estructura y el movimiento de la empresa.

2. Una vez construido el grupo focal y por el método de lluvia de ideas se referencia las fortalezas y debilidades más significativas de la empresa en el área contable.

3. Después de hacer el referenciación de los problemas se hace un tamizaje de esos problemas para simplificar y reducir los inconvenientes más significativos.

4. Posterior a esto cada integrante del grupo focal realiza una calificación el peso relativo de cada problema.

5. Se realiza una ponderación de cada uno de los problemas para depositarlos en la matriz 
Cuadro 1. Matriz EFI SYSCON

\begin{tabular}{|l|l|l|l|}
\hline FORTALEZAS & CALIFICACION & PORCENTAJE & RESULTADO \\
\hline prestados & & $40 \%$ & \\
\hline $\begin{array}{l}\text { Amplio conocimiento de la } \\
\text { materia }\end{array}$ & 5 & $30 \%$ & 2 \\
\hline Fidelidad de los clientes & 4 & & 1,5 \\
\hline Total & 5 & $30 \%$ & 1,2 \\
\hline DEBILIDADES & & $\mathbf{1 0 0 \%}$ & $\mathbf{4 , 7}$ \\
\hline Falta de Orden & 3 & $30 \%$ & 0,9 \\
\hline Falta de implementación de & 4 & $50 \%$ & 2 \\
\hline un manejo adecuado de & & & \\
inventario & & $\mathbf{1 0 0 \%}$ & $\mathbf{3 , 7}$ \\
\hline Falta de agilidad en el & 4 & & 0,8 \\
\hline servicio & & & \\
\hline Total & & & \\
\hline
\end{tabular}

Fuente: elaboración propia, basado en la investigación realizada en la empresa SYSCON 


\section{Resumen de la practica}

El estudio está dirigido principalmente al manejo de los inventarios dentro de la empresa SYSCON LTDA, en el cual se ha verificado que presenta varias dificultades en el desarrollo de separar los costos de la empresa con los costos de inventarios.

Para llevar a cabo el desarrollo de la misma, se hizo necesario realizar observaciones en la empresa para determinar donde se encuentran dichas dificultades, también se hizo revisión de los documentos de los sistemas contables, también se habló con la persona encargada de este proceso y es quien está encargada del desarrollo de esta actividad.

Así se llegó a la conclusión de que en el interior de la empresa no se está implementando de buena manera los inventarios, no se está desarrollando bien el programa para esta actividad y por lo tanto esto genera un desorden a la hora de tener un control sobre ellos.

\section{Planteamiento del problema}

De acuerdo al estudio que se ha realizado en el manejo de los inventarios, se evidencia que la empresa SYSCON LTDA presenta unas falencias en torno a este, pese a que su actividad principal es la prestación de servicio por medio de software, no se le está brindando la suficiente importancia al manejo de los inventarios, lo que no permite tener un control adecuado sobro ello.

Por esto, es necesario que la empresa empiece a implementar controles para organizar y controlar mejor los costos de inventarios y así mejorar los procesos contables. 


\section{Alternativa de solución}

Mejorar la recepción de los inventarios: Ayudaría a optimizar el tiempo a la hora de buscar los costos de inventario de cada servicio, tendría un impacto positivo para la empresa porque evitaría confusiones a la hora de separar los diferentes costos a los que se incurre.

Capacitar al personal encargado para el manejo de los inventarios: Esta función ayudaría a mejorar el control de los costos de inventario, tendría un impacto positivo para la empresa ya que la persona encargada de la contabilidad podrá separar y ayudar mejor en la toma de decisiones a la hora de brindar o prestar un servicio.

Codificar todos los costos de inventario: Controlaría mejor los costos que son atribuibles a la empresa, pero traería un impacto negativo ya que al tener unos servicios anteriormente prestados no se podría tener un dato exacto de los costos a de inventario a los que se ha incurrido antes, así que la información quedaría incompleta.

\subsection{Selección de la Alternativa}

El manejo de los inventarios en la empresa SYSCON LTDA es de vital importancia, además tiene un gran impacto de las ganancias que genera la empresa, por este motivo es fundamental analizar y plantear actividades para el mejoramiento y separación de los inventarios, en lo que concierne en el adecuado manejo de los costos ya sean de inventario o los que incurre la empresa, realizándose un manual el cual funcione como el control de los inventarios, guie al personal a cargo, evitando tener la confusión de que son y no son inventarios y así obtener mejores resultados en el manejo de la misma. 


\section{Plan de acción.}

En este apartado se referenciarán las diferentes actividades, propósitos, tiempos y responsables que se deben implementar par que exista un buen funcionamiento de la alternativa seleccionada

Cuadro 2. Plan de acción. SYSCON

\begin{tabular}{|l|l|l|l|l|l|}
\hline$N^{\circ}$ & Actividad & Propósito & Tstrategia & Responsable \\
\hline 1 & $\begin{array}{l}\text { Comprensión del } \\
\text { proceso }\end{array}$ & $\begin{array}{l}\text { Inicialmente se necesita que la } \\
\text { persona encargada de este } \\
\text { proceso tenga el conocimiento de } \\
\text { lo que se pretende hacer. }\end{array}$ & $\begin{array}{l}\text { Crear una estrategia y tener } \\
\text { claro el objetivo y el objetivo } \\
\text { al cual se desea llegar con este } \\
\text { nuevo planteamiento }\end{array}$ & 2 días & Área administrativa \\
\hline 2 & Mejorar el Proceso & $\begin{array}{l}\text { Reorganizar y estructurar el nuevo } \\
\text { proceso a implementar }\end{array}$ & $\begin{array}{l}\text { Organizar el manual que se } \\
\text { tiene y hacer las mejoras } \\
\text { necesarias para detallar cada } \\
\text { proceso }\end{array}$ & Área administrativa \\
\hline 3 & $\begin{array}{l}\text { Mejoramiento } \\
\text { Continuo }\end{array}$ & $\begin{array}{l}\text { Mejorar y así la empresa tiene un } \\
\text { crecimiento adecuado a sus } \\
\text { necesidades }\end{array}$ & $\begin{array}{l}\text { El mejoramiento es algo que } \\
\text { se plantea a la empresa a } \\
\text { pesar que es una actividad que } \\
\text { se practica todos los días }\end{array}$ & Todos los días laborados & Área Administrativa \\
\hline
\end{tabular}

Fuente: elaboración propia, basado en la investigación realizada en la empresa SYSCON 


\section{Resultados de la práctica.}

Con la ejecución de la practicas profesionales al interior de la empresa SYSCON LTDA y después de analizar el problema que actualmente presenta, se logró determinar que su principal inconveniente es no contar con un correcto manejo y control de los inventarios, el cual permita de una manera eficiente sacar unos mejores costos y acoplarse perfectamente a la empresa, dada esta situación, se ve viable implementar un manual de control de inventarios, que guie a la persona encargada de esta labor en su manejo y a su vez optimizar la administración de recursos en cuanto a los costos de servicio que permitan tomar decisiones más eficientes. El diagnostico también permitió generar alternativas de mejoramiento al interior de la empresa.

Finalmente, me queda una experiencia muy agradable la cual me ha permitido desarrollar conocimientos y habilidades los cuales he obtenido durante el proceso de formación profesional en el área de contaduría pública, los cuales han sido base para el desarrollo de esta. Así mismo lo que se ha buscado en el proceso, ha sido rodearme en el área contable y poder, poder profundizar en el conocimiento para desempeñar la practica durante el periodo de formación, también tener la experiencia de trabajar en equipo y poder enfrentar los diferentes problemas y dificultades que se presentan en el área laboral. 


\section{Conclusiones}

De acuerdo con el tema abordado a lo largo de esta investigación, se puedo observar la importancia de tener un buen manejo y control de los inventarios, ya que de estos depende básicamente las utilidades que genere la compañía.

Con el fin de realizar una búsqueda más rigurosa, dirigida al manejo y control que se tiene sobre los inventarios, se hizo necesario inicialmente inspeccionar todo lo relacionado con los costos de los servicios que ha brindado la empresa, ya que esta es la parte más importante para el desarrollo económico de la misma y por ende resulta de vital importancia un seguimiento adecuado, ya que nos brinda una capacidad de predicción y nos permite mantener un equilibrio entre lo que se hace y lo que ingresa. Bajo estas circunstancias se hace evidente la necesidad que presenta la compañía por mejorar el manejo y el control que se le ha venido dando a este.

Como información adicional, el método usado para la recolección de información, como observación del proceso y la interacción del personal que lo operan, fueron el medio directo para dar inicio a la elaboración del objetivo principal al que apunta la investigación y por ende al planteamiento del problema que presenta el proceso actual de la empresa SYSCON LTDA. Con dicha investigación podemos concluir que el método utilizado no es el más eficiente y claro, se consideró de vital importancia la estructuración de un manual enfocado en el proyecto principal del proyecto ya que por falta de un control adecuado dificultan las diversas operaciones.

Finalmente, se realizaron las propuestas adecuadas para el mejoramiento de los puntos débiles que encontraron durante la recolección de la información. Por el resto de las cosas está en proceso de mejoramiento y requiere del compromiso no solo del área 
encargada, sino también del área administrativa, ya que sin esta responsabilidad no se podrá mantener estas mejoras.

\section{Recomendaciones.}

A continuación, se propondrán una serie de recomendaciones con el fin de que la propuesta realizada para mejorar los procesos de control de inventarios se practique de forma eficiente.

- Implementar un control que permita mejorar la actividad que se cumple en la empresa.

- Mantener un orden en los costos de los inventarios, el cual permita identificar de forma eficaz al momento de prestar el servicio.

- Reportar las novedades sobre cualquier asunto que comprometa y se encuentre directamente ligado con el proceso de los inventarios.

\section{Lecciones aprendidas.}

1. Una de las lecciones más importantes que me dejo las prácticas empresariales es el correcto manejo que se le da al aprendizaje con respecto al mundo laboral al que salimos a enfrentarnos todos los estudiantes.

2. Aprendí hacer análisis de los diferentes errores que se cometen en las empresas, sea humano o por computador, ya que al ser algo con poca relevancia como lo son los costos por inventarios en las empresas de servicio se deja de recibir rentabilidades superiores. 
3. Resolver problemas que se presentan día a día en la labor desempeñada y la importancia del trabajo en equipo

\section{Bibliografía}

Ballou, R. (2004). Logística, administración de la cadena de suministro. En R. H. Ballou, Logística, administración de la cadena de suministro (págs. 550-760). México: Pearson.

Chopra, S., \& Meindl, P. (2008). Administración de la cadena de suministro . En S. Chopra, \& P. Meindl, Administración de la cadena de suministro (págs. 50-51). México: Pearson Educación.

DINERO. (10 de Febrero de 2009). Obtenido de S.A.S el tipo de sociedad más usado en Colombia: https://www.dinero.com/negocios/articulo/sas-tipo-sociedad-usadocolombia/84554

DINERO. (23 de 07 de 2009). Revista Dinero. Obtenido de Dinero.

Enrique Benjamín, F. (2009). Organización de empresas. En F. Enrique Benjamín, Organización de empresas (pág. 124). México: Mc Graw Hill.

Gayle, R. (1999). Contabilidad y administración de costos. México: McGraw-Hill Interamericana.

Guerrero, H. (2009). Inventario, manejo y control. En H. Guerrero, Inventario, manejo y control. Colombia: Ecoe ediciones.

Herrera, J. D. (11 de Mayo de 2016). Actualicese. Obtenido de https://actualicese.com/actualidad/2016/05/11/inventario-de-prestadores-deservicios-segun-normas-internacionales-para-pymes/

Kotschevar, L., \& Tanke, M. (1991). Managing Bar and Beverage Operations. Instituto Educativo de American Hotel \& Mot.

Ortiz, M. (2004). Procedimiento para la gestión de inventarios de empresas comerciales y de servicios. Tesis Doctoral. Cuba: Universidad de la Habana.

Sallenave, J. (2002). La gerencia integral ¡No le tema a la competencia, témale a la incompetencia! . En J. P. Sallenave, La gerencia integral iNo le tema a la competencia, témale a la incompetencia! (pág. 280). Bogotá: Norma.

Sucky, E. (2005). Inventory management in supply chains. En E. Sucky, Inventory management in supply chains (págs. 252-253). Estados Unidos. 
Wild, T. (2002). Mejores prácticas en gestión de inventarios. En T. Wild, Mejores prácticas en gestión de inventarios.

\section{Anexos}

\section{Fotografías:}

- Primera Visita

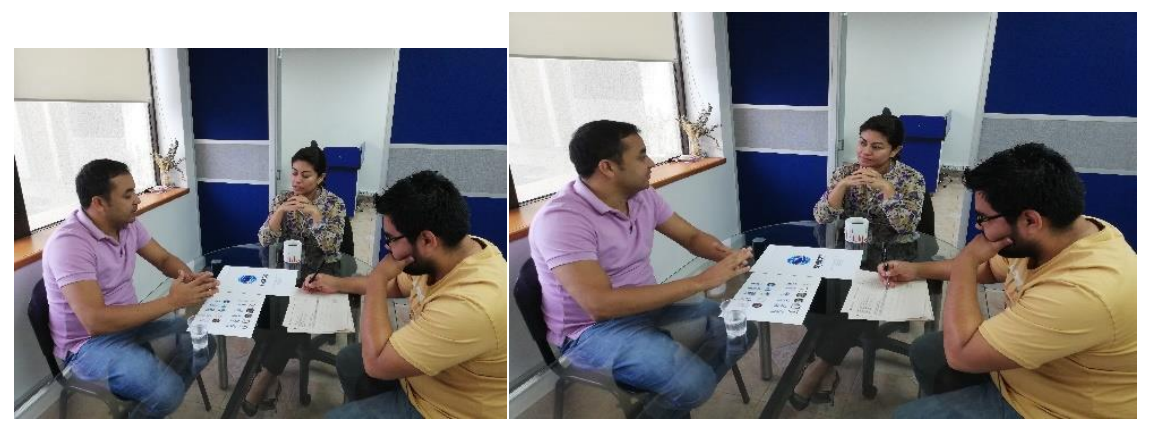

- Segunda Visita

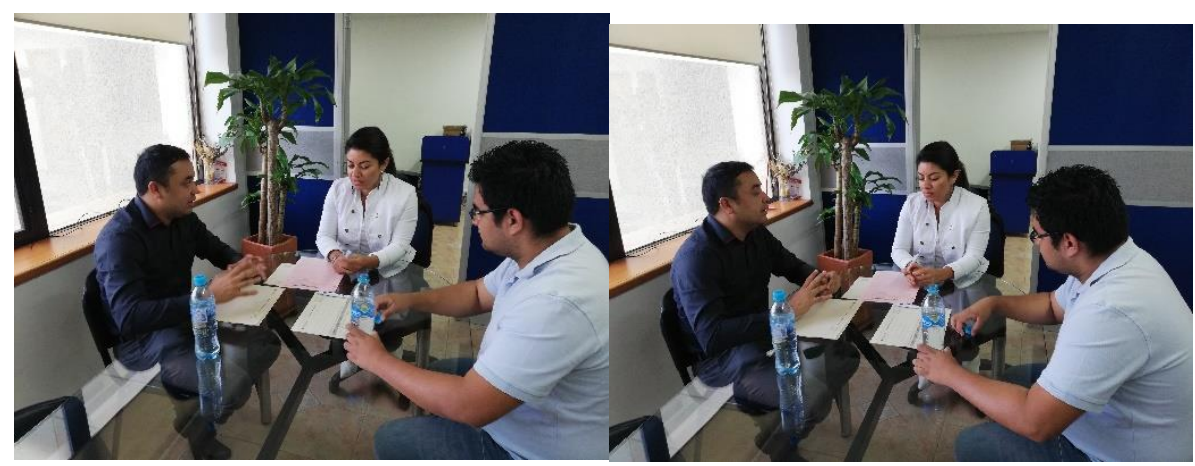


- Visita de cierre.

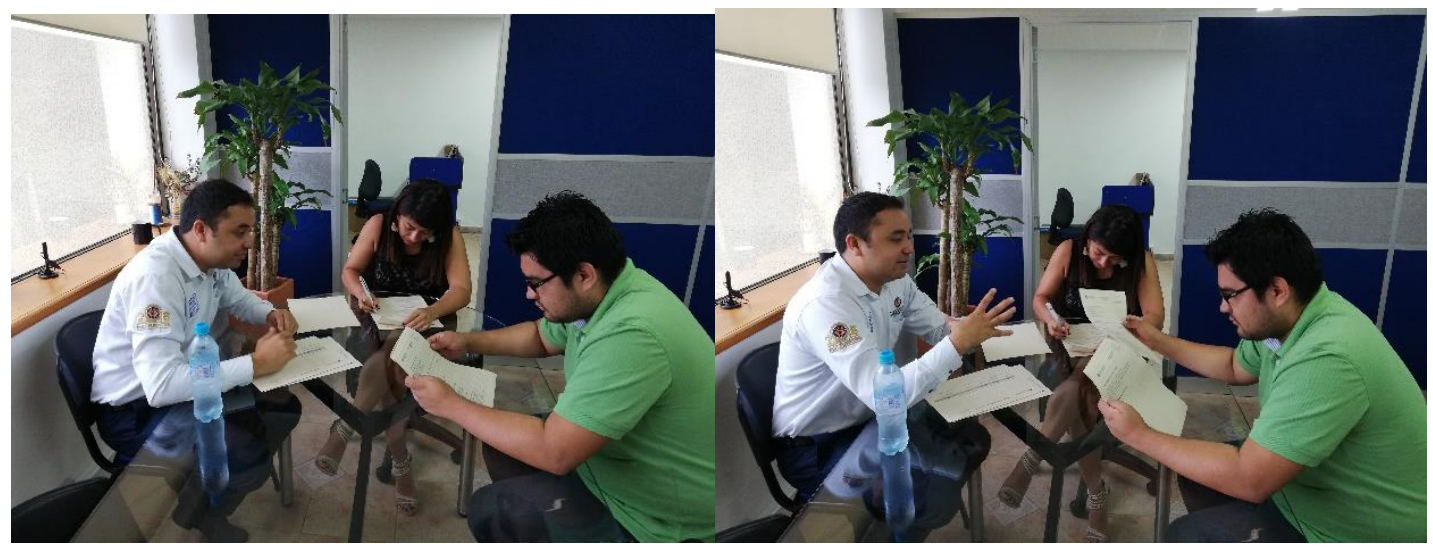

